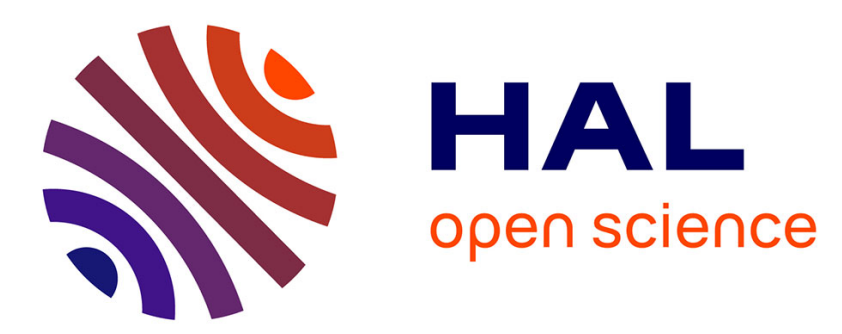

\title{
Asymptotic properties of incoherent waves propagating in an all-optical regenerator line
}

\author{
Stéphane Pitois, Christophe Finot, Lionel Provost
}

\section{To cite this version:}

Stéphane Pitois, Christophe Finot, Lionel Provost. Asymptotic properties of incoherent waves propagating in an all-optical regenerator line. Optics Letters, 2007, 32 (22), pp.3263-3265. 10.1364/OL.32.003263 . hal-00406184

\section{HAL Id: hal-00406184 \\ https://hal.science/hal-00406184}

Submitted on 28 Apr 2010

HAL is a multi-disciplinary open access archive for the deposit and dissemination of scientific research documents, whether they are published or not. The documents may come from teaching and research institutions in France or abroad, or from public or private research centers.
L'archive ouverte pluridisciplinaire HAL, est destinée au dépôt et à la diffusion de documents scientifiques de niveau recherche, publiés ou non, émanant des établissements d'enseignement et de recherche français ou étrangers, des laboratoires publics ou privés. 


\title{
Asymptotic properties of incoherent waves propagating in an all-optical regenerators line
}

\author{
Stéphane Pitois and Christophe Finot \\ Institut Carnot de Bourgogne (ICB), UMR-CNRS 5209, Université de Bourgogne, \\ 9 Avenue Alain Savary, 21078 Dijon, France
}

\begin{abstract}
Lionel Provost
Optoelectronics Research Centre (ORC), University of Southampton, Southampton SO17 1BJ, United-Kingdom
\end{abstract}

In this work, we present an original method to generate optical pulse trains with random timeinterval values from incoherent broadband sources. More precisely, our technique relies on the remarkable properties of a line made of cascaded SPM-based optical regenerators. Depending on the regenerator parameters, various regimes with noticeably different physical behaviors can be reported.

OCIS codes: (190.5530) Pulse propagation and solitons; (190.3100) Instabilities and chaos; (070.4340) Nonlinear optical signal processing; (320.7140) Ultrafast processes in fibers. 


\section{Introduction}

Over the past years, optical transmission systems enabling very high bit rate operations (160 Gbit/s and beyond) have undoubtedly focused much attention from many research groups. Alleviating the absence of high-bandwidth electronics at such repetition rates, all-optical techniques have been proposed as a viable alternative solution for signal processing. In particular, all-optical fiber-based regenerators relying on four-wave mixing [1], self-phase modulation (SPM) [2], non-optical loop mirrors optical switch [3] or similariton formation [4] have been reported. In essence, optical regenerators allow discrimination between high power signal and low power noise thanks to the Kerr nonlinearity, whereas dissipation allows useless optical energy to be rejected from the system. Among the various regenerators reported in the literature, the scheme introduced by Mamyshev in Ref. [2] appears as the most robust to simultaneously offer noise suppression in empty bitslots and power equalization on the ones pulses of return-to-zero sequences. Basically, the principle of the Mamyshev's optical Regenerator (MR) relies on the SPM-induced spectral broadening of the degraded signal followed by a subsequent offset spectral filtering. Given the ease of implementation, numerous works have focused their attention on the performance of such regenerators to efficiently restore the quality of corrupted data streams for telecommunication applications [2, 5-7].

In this work, we adopt a rather different perspective which allows us to highlight original and fundamental properties of MRs. More precisely, we study the long-term evolution of light by considering the propagation in a line made of a large number of concatenated double-stage MRs, thus focusing our attention on the asymptotic resulting properties of such an arrangement. In a first part, we describe the regenerator line under consideration and report the existence of 
particular eigenpulses preserving both phase and intensity profiles along the line. In a second part, we take benefit of this remarkable property to generate randomly distributed pulse trains from an incoherent light source. Finally, for some suitable sets of parameters, we demonstrate that the cascaded MRs can support multiple eigenpulses and even limit-cycles.

\section{Principle}

The system under consideration (Fig. 1a) is formed by the concatenation of $\mathrm{N}$ identical elementary optical blocks. Each elementary block comprises two distinct MR stages. In a first time, light undergoes a large spectral broadening in a highly nonlinear normally-dispersive optical fiber due to the SPM. A detuned optical band-pass Gaussian optical filter (OBPF 1 in Fig. 1a), centered around $\omega_{0}+\Omega$, is then employed to carve into the spectrum. The shift of the OBPF central frequency washes out from the system a large part of the energy. As a result, only high peak power pulses which have undergone spectral broadening during the propagation can transmit a part of their energy after filtering. The system is thus intrinsically strongly dissipative. An intermediate optical amplifier compensates for the loss inherent to the described process and boosts the output power to an adequate level for the next MR. The second stage operates in a similar way as the first stage, except that the optical filter (OBPF 2 in Fig. 1a) is now centered around the initial frequency $\omega_{0}$ and ensures a transparent operation for the whole optical block [8]. Following the proposed implementation, evolution of light along the optical system was modelled by numerically solving the following nonlinear Schrödinger equation :

$$
\frac{\partial u}{\partial z}+\frac{i}{2} \beta_{2} \frac{\partial^{2} u}{\partial t^{2}}=i \gamma|u|^{2} u
$$


where $u$ is the slowly-varying envelope of the electric field, $\beta_{2}$ is the second-order dispersion parameter and $\gamma$ the nonlinear coefficient. For the sake of simplicity, we do not incorporate noise from the amplifier and fiber losses in our model.
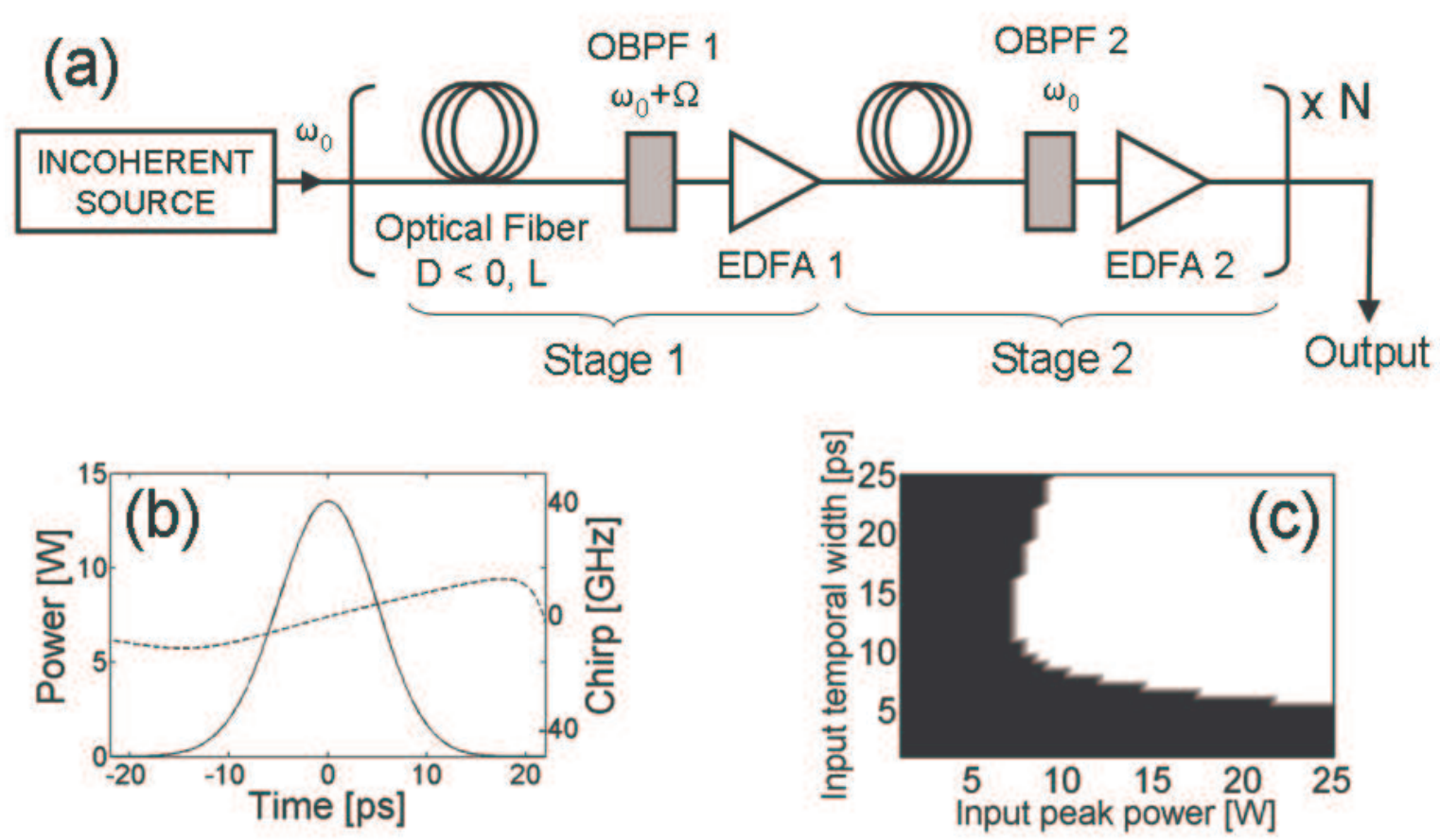

Fig. 1. (a) Schematic diagram of the regenerator line. OBPF : Optical Band-Pass Filter, EDFA : Erbium Doped Fiber Amplifier. (b) Eigenpulse intensity (solid line) and phase (dashed line) profiles for configuration P1. (c) Asymptotical state of a Gaussian pulse propagating in the line as a function of its initial width and peak power. White-shaded area: the pulse converges towards the eigenpulse, black-shaded area: the pulse is attenuated and finally suppressed.

\section{Existence of eigenpulses}

Before studying the propagation of an incoherent wave in the regenerator line, some physical insights could be gained by considering the asymptotic evolution of a single Gaussian pulse in the system. For instance, we consider a chain comprising regenerators of which parameters 
follow the configuration P1 from Table I. Following the results from Ref [5], this configuration corresponds to a monotonic transfer function exhibiting a constant output power (plateau) over a large input power range.

TABLE I : Parameters of the different configurations

\begin{tabular}{|c|c|c|c|c|}
\hline Configuration & Fiber Length L & Dispersion D & Frequency Offset $\Omega$ & EDFA Gain G \\
\hline P1 & $1240 \mathrm{~m}$ & $-5 \mathrm{ps} / \mathrm{nm} / \mathrm{km}$ & $150 \mathrm{GHz}$ & $10.6 \mathrm{~dB}$ \\
\hline P2 & $413 \mathrm{~m}$ & $-5 \mathrm{ps} / \mathrm{nm} / \mathrm{km}$ & $80 \mathrm{GHz}$ & $7 \mathrm{~dB}$ \\
\hline P3 & $2000 \mathrm{~m}$ & $-0.8 \mathrm{ps} / \mathrm{nm} / \mathrm{km}$ & $100 \mathrm{GHz}$ & $9 \mathrm{~dB}$ \\
\hline P4 & $1000 \mathrm{~m}$ & $-0.5 \mathrm{ps} / \mathrm{nm} / \mathrm{km}$ & $90 \mathrm{GHz}$ & $9 \mathrm{~dB}$ \\
\hline
\end{tabular}

For this configuration and depending on the characteristics of the input pulse (full width at halfmaximum temporal width and peak power), we found that a Gaussian pulse propagating in the regenerator chain can converges towards two well-defined states. The first state corresponds to the absence of light, demonstrating that the propagating pulse is progressively attenuated, and eventually completely suppressed. The second state corresponds to a pulse of determined intensity and phase profiles (see Fig. 1b). More interestingly, we observe that this particular pulse is left unaffected after propagation into an elementary block. Consequently, this stable solution is denoted as eigenpulse by a direct analogy with linear algebra. It is worth noting that the eigenpulse shape and width are mainly imposed by the filter shape and bandwidth [6], whereas the spectral chirp results from the nonlinear propagation along the optical fibers [5]. The convergence dynamics towards the two possible states is illustrated in Fig. 1c in which basins of attraction corresponding to the system have been plotted as a function of the initial parameters of the input Gaussian pulse. The white-shaded area corresponds to an attraction towards the 
eigenpulse of the line whereas the dark-shaded area represents the state for which pulse is suppressed.

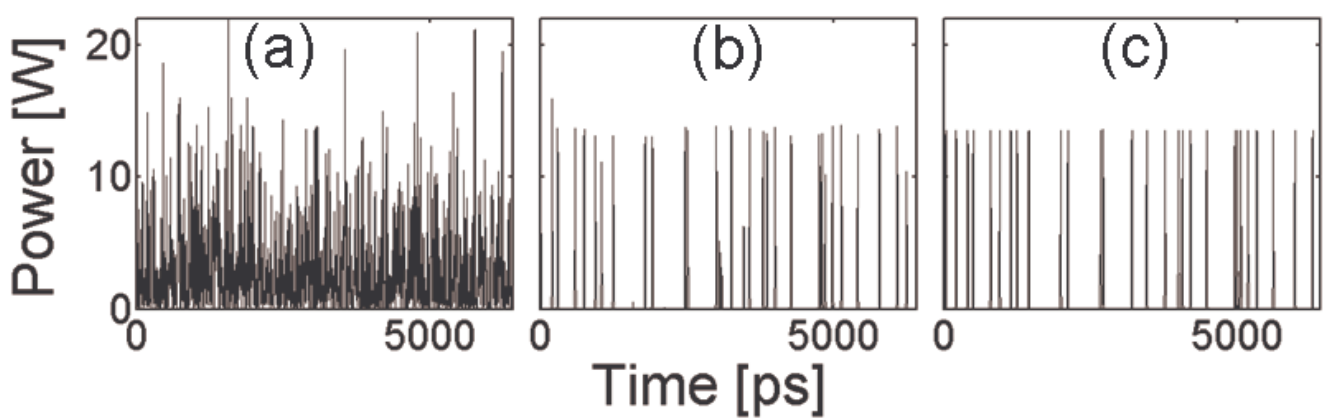

Fig. 2: Evolution of an incoherent wave with the parameters of configuration P1. (a) input, (b) for $N=2,(c) N=30$.

\section{Generation of random-time pulses sequences}

We consider now the evolution of an incoherent wave which slowly varying amplitude is assumed to be a stochastic field of zero mean, obeying Gaussian statistics. The spectral bandwidth of this incoherent source was fixed to $80 \mathrm{GHz}$, corresponding to a coherence time in the order of 12.5 ps. From an intuitive point of view, we can expect that the propagation of such a multiple peaked structure in the line would asymptotically result in the vanishing of the portion of the signal exhibiting small peaks, whereas convergence towards an eigenpulse can be anticipated for higher peaks. This process is illustrated in Fig. 2 where we have plotted the evolution of an incoherent input signal (Fig. 2a) when few cascaded stages were considered. As expected, and for only $\mathrm{N}=2$ (Fig. 2b), a sequence of picosecond pulses of nearly similar shape is observed. By increasing $\mathrm{N}$, we can furthermore check that each outgoing pulse matches the eigenpulse associated to the line (e.g. N=30, Fig. 2c). It is understandable that the number and 
the temporal localization of the output pulses are intrinsically seeded by the initial random process associated to the input incoherent signal, and thus they are highly erratic.

At this stage, we would like to emphasize that the operating regime of the optical regenerator, or equivalently the fiber parameters and the offset filter position, have a substantial influence on the asymptotic evolution of the propagating input signal. To illustrate this point, we consider the parameters of the configuration P2 given in Table I. This regenerator line approximately exhibits the same eigenpulse as the configuration P1 but differs by a different power transfer function with a non-monotonic evolution. As a result, the basin of attraction obtained for an incident Gaussian pulse differs significantly from the one obtained with the first configuration (Fig. 3a): we observe the existence of two distinct narrow areas to be compared with a much larger domain for configuration P1. Consequently for the same input incoherent wave, a reduced number of pulses is generated, as illustrated in Fig. 3c.

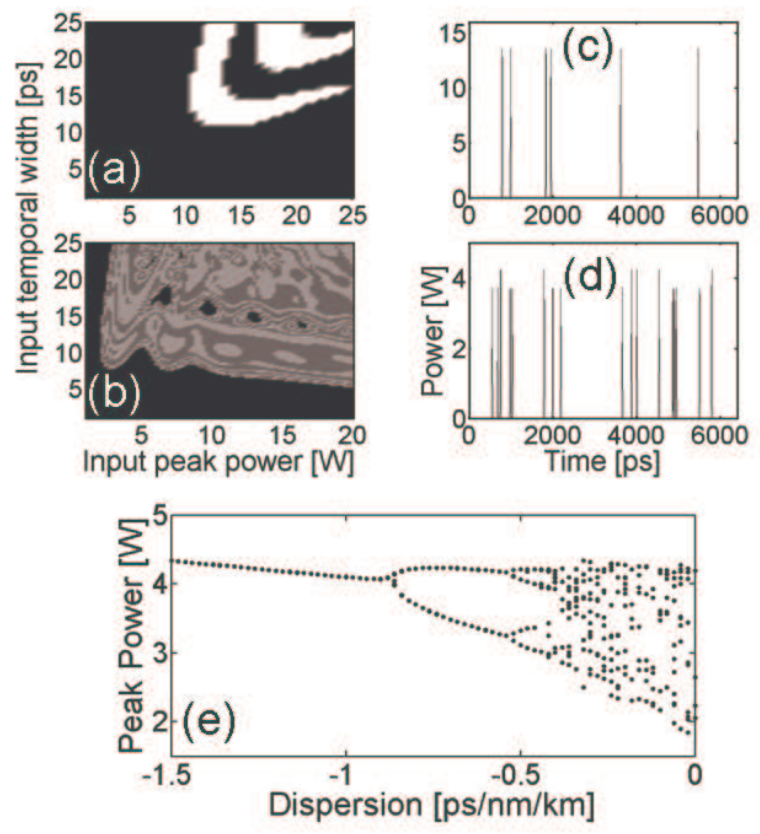


Fig. 3. (a) and (b) Asymptotical states of a Gaussian pulse propagating in the line as a function of its initial width and peak power for configurations P2 and P3. (a): White-shaded area: the pulse converges towards the eigenpulse; black-shaded area: the pulse is attenuated and finally suppressed. (b): White-shaded area: the pulse converges towards the second eigenpulse, grayshaded area: the pulse converges towards the first eigenpulse and for black-shaded area: the pulse is attenuated and finally suppressed. $(c)$ and $(d)$ : Asymptotic pulse trains for configurations $P 2$ and P3. (e): Evolution of the different eigenpulse peak powers as a function of the dispersion parameter D for the configuration P3.

\section{Multiple eigenpulses and limit-cycles}

For other suitable sets of parameters, we have also observed that the regenerator line reveals more complex behaviors, such as the existence of multiple eigenpulses and limit-cycles. For instance, looking at the evolution for the configuration P3 in Table I, two distinct eigenpulses coexist in the system with respective peak powers of $3.7 \mathrm{~W}$ and $4.2 \mathrm{~W}$. When considering an input Gaussian pulse, the convergence towards one of the two invariant solutions or towards a suppressed state is highly dependent on the input pulse characteristics as depicted in the contour plot in Fig. 3b. We observe a multiplicity of basins of attraction for the three possible states forming a complex pattern associated with the formation of the $3.7 \mathrm{~W}$ peak power eigenpulse (gray-shaded areas), or $4.2 \mathrm{~W}$ peak power eigenpulse (white-shaded areas), or alternatively to the absence of light (black-shaded areas). Similarly when an input incoherent wave is considered, an asymptotical evolution towards a pulse stream exhibiting a random combination of the two eigenpulses is observed (see Fig. 3d). 
At this point, we would like to emphasize that the number of attractors, and thus the possibility to observe chaos in the system, depends on the ratios between the different parameters defining the system. To illustrate this property, we consider now the configuration P3 and study the long term evolution of a given incoherent wave for different values of the dispersion parameter D ranging between $-1.5 \mathrm{ps} / \mathrm{nm} / \mathrm{km}$ and $0 \mathrm{ps} / \mathrm{nm} / \mathrm{km}$. The peak power values of the multiple eigenpulses that coexist in the system are reported in Fig. 3e as a function of the dispersion parameter D. For high dispersion values, the system converges towards a single well-defined eigenpulse. As the dispersion decreases, many bifurcations occur and the number of eigenpulses significantly increases. Finally, for very small values of D, a large number of attractors coexist and the system presents a chaotic behaviour.

Now, we would like to report a particular case for which a propagating pulse can evolve following a limit-cycle trajectory comprising a plurality of eigenpulses. Taking for example the parameters of the configuration $\mathrm{P} 4$ from Table I, we demonstrate that the corresponding cascaded MRs can support a limit-cycle made of three eigenpulses respectively denoted Pulse A, B, and C plotted in Fig. 4a-c. After each elementary MRs block, an A-type input pulse is transformed into a B-type, a B-type into a C-type, and eventually a C-type into an A-type, so that the limit-cycle takes the form: [Pulse A $\rightarrow$ Pulse $\mathrm{B} \rightarrow$ Pulse $\mathrm{C} \rightarrow$ Pulse A $\rightarrow$...]. It is worth noting that a longer limit-cycle made of four elements can be also observed for the same configuration.

When an incoherent input wave is injected in this system, the two co-existing limit-cycles promote the apparition of a stream made of multi-level amplitude pulses which is function of the number N. This behaviour is represented in Fig. 4 which shows the evolution of an initial 
incoherent wave (Fig. 4d) into a sequence of pulses with multiple peak powers (Fig. 4e and 4f). Note the difference in the sequence when an additional elementary block is added.
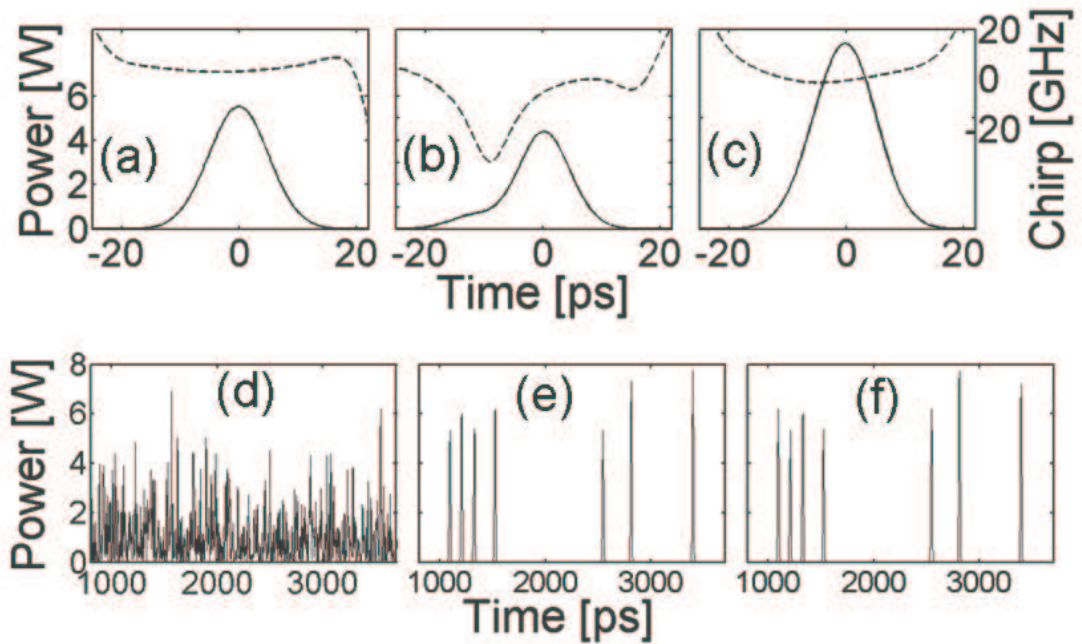

Fig. 4. (a), (b), and (c) represent intensity and phase profiles of the pulses constituting the limitcycle. The pulses peak powers are $5.51 \mathrm{~W}, 4.39 \mathrm{~W}$, and $8.35 \mathrm{~W}$, respectively. (d) Input incoherent sequence. (e) Pulse train for $\mathrm{N}=299$ trips and (f) pulse train for $\mathrm{N}=300$.

\section{Conclusion}

In this work, we have used a novel approach to highlight original properties of all-optical regenerators based on self-phase modulation and subsequent spectral filtering. It results that such regenerators can be used to generate random sequences of optical pulses from an incoherent wave. This behaviour, which could find applications in the field of random processes generation, is related to the existence of eigenpulses which can propagate unchanged through the regenerator line and act as attractors for the incoming pulses. We anticipate that this last property could be of 
a particular interest for the design and optimisation of double-stage Mamyshev's optical regenerators for optical telecommunications.

By changing the regenerator parameters, we also report the existence of multiple eigenpulses and limit-cycles. Finally, we expect from these results that Mamyshev's regenerators are likely to act as efficient nonlinear gates in fiber cavity lasers, and thus become a novel method to achieve passive mode-locking.

We would like to acknowledge Dr. P. Petropoulos and Prof. D.J. Richardson (Optoelectronics Research Centre, Southampton, UK) for fruitful discussions. 


\section{References including full titles:}

1. E. Ciaramella, and S. Trillo, "All-optical signal reshaping via four-wave mixing in optical fibers.," IEEE Photon. Technol. Lett. 12, 849 (2000).

2. P. V. Mamyshev, "All-optical data regeneration based on self-phase modulation effect," in European Conference on Optical Communication, ECOC'98 (Institute of Electrical and Electronics Engineering, Madrid, Spain, 1998), pp. 475-476.

3. N. J. Doran, and D. Wood, "Nonlinear-optical loop miror," Opt. Lett. 13, 56-58 (1988).

4. C. Finot, S. Pitois, and G. Millot, "Regenerative 40-Gb/s wavelength converter based on similariton generation," Opt. Lett. 30, 1776-1778 (2005).

5. L. Provost, C. Finot, K. Mukasa, P. Petropoulos, and D. J. Richardson, "Design scaling rules for 2R-Optical Self-Phase Modulation-based regenerators 2R regeneration," Opt. Express 15, 5100-5113 (2007).

6. M. Rochette, L. B. Fu, V. G. Ta'eed, D. J. Moss, and B. J. Eggleton, "2R optical regeneration : an all-optical solution for BER improvement," IEEE J. Select. Top. Quantum Electron. 12, 736-744 (2006).

7. J. Leuthold, G. Raybon, Y. Su, R. J. Essiambre, S. Cabot, J. Jacques, and M. Kauer, "40 Gbit/s transmission and cascaded all-optical wavelength conversion over $1000000 \mathrm{~km}$," Electron. Lett. 38, 890-891 (2002).

8. M. Matsumoto, "Efficient all-optical 2R regeneration using self-phase modulation in bidirectional fiber configuration," Opt. Express 14, 11018-11023 (2006). 


\section{References without titles:}

1. E. Ciaramella, and S. Trillo, IEEE Photon. Technol. Lett. 12, 849 (2000).

2. P. V. Mamyshev, in European Conference on Optical Communication, ECOC'98 (Institute of Electrical and Electronics Engineering, Madrid, Spain, 1998), pp. 475-476.

3. N. J. Doran, and D. Wood, Opt. Lett. 13, 56-58 (1988).

4. C. Finot, S. Pitois, and G. Millot, Opt. Lett. 30, 1776-1778 (2005).

5. L. Provost, C. Finot, K. Mukasa, P. Petropoulos, and D. J. Richardson, Opt. Express 15, 5100-5113 (2007).

6. M. Rochette, L. B. Fu, V. G. Ta'eed, D. J. Moss, and B. J. Eggleton, IEEE J. Select. Top. Quantum Electron. 12, 736-744 (2006).

7. J. Leuthold, G. Raybon, Y. Su, R. J. Essiambre, S. Cabot, J. Jacques, and M. Kauer, Electron. Lett. 38, 890-891 (2002).

8. M. Matsumoto, Opt. Express 14, 11018-11023 (2006). 